\title{
Carmen Rosa Rea Campos, Cuando la otredad se iguala. Racismo y cambio estructural en Oruro- Bolivia, México, El Colegio de México, 2015, 505 pp.
}

Reseña por José Eduardo Calvario Parra, Fernanda Elizalde Castillo*

Perfiles Latinoamericanos, 26(52) $\mid 2018$ DoI: $10.18504 / \mathrm{pl} 2652-018-2018$

\section{E}

tema del racismo es importante sin lugar a dudas en la historia regional latinoamericana en cuanto a los conflictos, luchas, cambios culturales, procesos de exclusión social y circunstancias sociohistóricas que algunas áreas geográficas de América han experimentado (Castellanos \& Landázuri, 2012). El libro de Rea Campos que aquí se reseña es producto de la tesis de doctorado "Oruro cholos totales". Racismo y crisis de identidades en la Bolivia contemporánea: el caso de la Ciudad de Oruro desarrollada por la autora en El Colegio de México. Desde el principio, el proyecto de investigación sobresalió por su empeño en abordar el problema endemoniado, como diría Norbert Elías (2009 [1977]), de la estructura y la agencia mediante un estudio empírico; y sobre todo por su agudeza al reformular la cuestión de la racialización en Bolivia en claro diálogo con autores de la cuestión.

Es un texto interesante no solo desde el punto de vista académico y científico sino porque también contribuye a reactualizar un tema que en la vida diaria es tratado de manera colateral y superflua e incluso con cierta jocosidad en los temas de sobremesa. Los chistes sobre la condición indígena, las canciones que evocan el color de piel en un tono esencialista, el manejo noticioso sobre la adjudicación de estereotipos racializadores, etc., son ejemplos de la actualidad del libro. La obra consta de 505 páginas que se dividen en seis capítulos, introducción y conclusiones.

* Doctor en Ciencia Social con especialidad en Sociología por El Colegio de México. Cátedras CONACYT-COLSON | josep.calvario@gmail.com

** Licenciada en Sociología por la Universidad de Sonora (México). Becaria de El Colegio de Sonora | ffernandaelizalde@gmail.com 
Es un trabajo que encuentra su valor en la distinción que la autora obtuvo como mejor tesis de la Academia Mexicana de Ciencias 2011, y en la rigurosidad de sus fuentes, y control teórico y metodológico al emplear los conceptos con cautela para no caer en lo que la Rea llama, recurriendo a Bourdieu, sociología espontánea. Sintéticamente se pueden mencionar cinco principales aportes de esta obra:

1. Es un avance del conocimiento al cuestionar, debatir y reformular las hipótesis dominantes respecto de la condición pluricultural y étnica de Bolivia y, sobre todo, de la prevalencia de la categoría de "mestizo" y/o la autoidentificación de la población en la dicotomía indígena versus no indígena.

2. En términos teóricos, supone una mirada sociológica enriquecida con el enfoque de Michel Foucault al entender el racismo como una técnica de poder que separa y divide a la población en dos o más partes, en la que una de ella se proclama representante de la "normalidad".

3. En términos metodológicos, se trata de una estrategia que combina y triangula técnicas de investigación cualitativas y cuantitativas. La conciliación y complementariedad de la técnica estadística y la entrevista no estructurada son llevadas a buen puerto. Rea toma la escuela como referente empírico en tanto espacio privilegiado para registrar disputas, rispideces, readecuaciones de significados socioculturales emergentes y dominantes.

4. Muestra la conexión entre los cambios estructurales, los cuales inician décadas anteriores al estudio, en especial, la modificación de la correspondencia entre la clase y la etnia, y las prácticas racializadoras en la vida cotidiana.

5. Es una contribución al debate en las ciencias sociales en cuanto a la definición del racismo, pues estimula la discusión con la perspectiva tradicional que ve al racismo exclusivamente como una forma ideológica de las clases dominantes sin entrever que es además un recurso de poder para ocultar las diferencias.

Básicamente, las aportaciones del libro se sitúan en la lógica de develar los mecanismos que re-producen el racismo con frecuencia desatendidos por estudiosos y estudiosas del racismo en América Latina. Por ejemplo, sobre la importancia del régimen político y el racismo, Rea pone a debate el vínculo directo y mecánico entre ambos. La tesis de que el régimen político es un factor clave para exacerbar o eliminar al racismo ha tenido acogida en sectores académicos latinoamericanos; otra de las ideas con cierta aceptación es la irreductibilidad del racismo y el nacionalismo. Sobre el primero, Rea señala que, por lo menos para el caso boliviano, no estuvo directamente vinculado con el sistema político de gobierno sino que más bien tuvo que ver con la lógica interna 
de procesos histórico-culturales en los que una élite económica se constituyó como proyecto "racial de mestizaje cultural". Sobre lo segundo, a lo largo de los seis capítulos se plasma el racismo visto como recurso de poder en términos macro y microsociales, y se refuerza la visión de que si la construcción de la identidad nacional, es decir, del proyecto de autodefinición nacional, se enlaza con lo racial este no lo determina per se sino que están en juego distintas variables sociológicas que posibilitan su reconstitución.

En este contexto, el libro de Rea representa un importante esfuerzo por situar, actualizar y discutir la cuestión racial, pero desde un punto de vista no esencialista, ahistórico y biológico, robustecido, eso sí, con herramientas teóricoconceptuales de las ciencias sociales.

En el primer capítulo, la autora nos da un recorrido sociohistórico por la formación de "una matriz de nación" en Bolivia mirando hacia la generación de otredades, en especial, en las disputas de las categorías raciales. El tema se discute en tres apartados: en el primero se aborda el periodo liberal, que va de fines del siglo XIX hasta mediados del xx, y que se expresa en lo que Rea denomina la biologización de las poblaciones indígenas y en el que toma vigor el discurso racial. El segundo apartado se ocupa del periodo nacionalista — segunda mitad del siglo xx- cuyo rasgo principal fue la racialización de las diferencias culturales con las que se da un impulso a las políticas asimilacionistas vía el discurso del mestizaje. El tercer apartado expone el periodo neoliberal, el cual arranca en la década de 1980 con la aplicación de políticas de reconocimiento de las identidades indígenas, pero, al decir de la autora, con el persistente proceso de racialización. Esta periodización no es lejana de lo sucedido en México, sobre todo en lo relativo a las políticas asimilacionistas de los gobiernos posrevolucionarios (Korsbaek \& Sámano, 2007).

A partir del año 2000 se reconfiguraron en Bolivia las dinámicas de aceptación y rechazo en el imaginario político de la participación activa de indígenas, en especial, con Evo Morales y el Movimiento al Socialismo (MAS). Pero es un error afirmar, dirá la autora en los siguientes capítulos, que eso haya significado la atenuación en automático o la eliminación del racismo solo porque en términos simbólicos había llegado un presidente de extracción indígena como lo es Morales.

En el segundo capítulo la autora se adentra en el importante debate nacional boliviano acerca del conteo estadístico de la condición étnica de la población, donde ella aprovecha para poner a prueba las afirmaciones sobre la condición mestiza por una parte, e indígena por la otra; lo hace a la luz de la aplicación de cuestionarios en escuelas de educación media superior de la ciudad de Oruro. Su hallazgo principal se refiere a que, en primer lugar, los criterios subjetivos como la autoidentificación en alguna de las categorías étnicas o racializadoras 
no se presentan con una identidad cultural homogénea sino que existe la flexibilidad en reconocerse no indígena y a la vez adscribirse como aymara o quechua; así también, aunque ciertamente la categoría de mestizo explica en parte la adscripción a una identidad cultural, dicha categoría no es trasparente ni homogénea. En segundo lugar, respecto al lugar de procedencia de los padres de los jóvenes entrevistados y de la lengua materna, Rea problematiza los argumentos que suponen que tales criterios objetivos definen la identidad étnica. Aunque sí existe una relación, señala la autora, esta es débil estadísticamente hablando; adscribirse como indígena, blanco y/o mestizo está mediado por el ingreso económico y profesión de los padres, por el efecto del lugar, y por otros elementos que más bien remiten, documenta Rea, a estrategias para evitar la exclusión o discriminación.

Los procesos de estigmatización y los signos raciales en la vida social boliviana son tratados en el tercer capítulo. Lo primero cumple la función de legitimar la dominación étnica y racial a pesar de que el sentido común remita a que en la actualidad en Bolivia, en Oruro, es imposible que se manifieste la discriminación étnica con categorías que racialicen al "otro". A ras de suelo, en discurso social, opera una ilusión de igualación. No obstante, y en el contexto de los cambios (en la estructura social sobre todo), ha permitido la resistencia y respuesta del sujeto racializado; ello se expresa en la confrontación simbólica entre el "indio de ayer" y el "indio de hoy". La estigmatización refleja la exigencia de que el "indio de hoy" vuelva a su cauce normal porque, como atestigua la investigación, una capa indígena importante ha accedido a mejores recursos sociales, simbólicos y materiales. El proceso de racialización pasa por las marcas, exclusivamente, del color de la piel, de la ocupación, de la vestimenta, del apellido, del origen, etc. En el caso del uso de la pollera (vestido) entre las mujeres aymaras o quechuas, esto es cargado simbólicamente de modo negativo, vinculándolo con la categoría históricamente elaborada de "chola". Este capítulo destaca por mostrarnos cómo el racismo se expande de modo centrifugado en distintas áreas de la geometría social y en diversos matices y áreas de influencia.

La idea de la ilusión de igualación puede ser utilizada no solo para entender este proceso sino que es posible trasladarlo a las relaciones de dominación masculina (empleando el famoso concepto del sociólogo francés Pierre Bourdieu). $\mathrm{Al}$ cruce entre "el indio de ayer" y el "indio de hoy" le antecede uno más, la situación de las mujeres aymara, las de ayer y las de hoy. Aunque no es el eje primario, con los datos que presenta Rea, así como con su análisis, es posible delinear la situación en términos de la exclusión-aceptación de las mujeres en los procesos de los que da cuenta la autora.

La gradación racial se analiza en el cuarto capítulo con particular distinción en cuanto al uso más preciso de este fenómeno como cadena de racialización en la 
cual Rea sostiene que no es en absoluto coherente, vertical, trasparente y simple. Por medio de tres estudios de caso, la autora muestra cómo el fenómeno racial se manifiesta cuando "los signos raciales no corpóreos son difusos" (p. 223), "el grado civilizatorio justifica las diferencias entre iguales étnicos" (p. 231) y existen "relaciones que enfatizan que siempre hay uno más inferior que el inferior" (p. 235). El material empírico es extraído de las entrevistas realizadas entre distintos informantes. Los relatos muestran parte de la vida cotidiana, los discursos y representaciones sociales sobre el ser "indio", y la distancia que se construye desde la igualación.

En el capítulo quinto, Rea muestra una fase ya no exclusivamente del espacio social cotidiano sino que rescata la dimensión institucional del proceso racializador, en particular, el del espacio educativo, espacio por excelencia de la reproducción social dirían Bourdieu \& Passeron (2008). El ámbito escolar representa un sitio privilegiado en el que aparecen desigualdades y procesos de exclusión mediante alguno de los ejes de distinción como la clase, el género o lo racial. La autora analiza los estereotipos que otrifican a los sujetos en diversas escuelas de Oruro. Primero las caracteriza señalando que la composición estudiantil se ha modificado; según Rea, la escuela en tanto institución educativa pasó de un sistema de relaciones cerrado a uno abierto, es decir, que tratándose de escuelas privadas la población que era de clase media blanqueada y definida no indígena, pasó a ser más heterogénea con el ingreso de indígenas o hijos de indígenas con alto capital económico. En los espacios escolares hay un sistema de diferenciación entre los estudiantes con varios criterios de desigualación: la apariencia física (fenotipo), y marcas sociales como los ingresos económicos, la ocupación laboral de los padres y el apellido, entre otros. Sin embargo, los criterios culturales, y no el capital económico, determina la clase social de los estudiantes. Según Rea, con el racismo se observan dos situaciones, la "normalidad" que es el racismo cotidiano, y la "perturbación", con la que se restablecen las condiciones de desigualdad social. La perturbación se aprecia con la presencia de estudiantes aymaras en las escuelas que antes eran de sistema cerrado; con ello se "supone un oscurecimiento e indianización" (p. 361) para los estudiantes de clase media blanqueada y definida no indígena, la cual se ha estancado económicamente, en tanto que los aymaras han aumentado su capital económico, es decir, que se han igualado en estos términos, aunque el dinero dejó de ser un elemento de distinción social y ahora son los rasgos faciales el principal indicador para establecer aquella diferenciación. Padres, estudiantes y profesores mantienen una relación tensa en la que se agrede todo aquello que es considerado "cholo"; los estudiantes pasan a estas relaciones tensas de cierre social consiguiendo así que el sujeto agredido opte por autoexcluirse. 
Estos hallazgos son relevantes si se observa el contexto de estratificación y desigualdad social en América Latina, y más aún cuando se replantean las miradas de los fenómenos y dinámicas socioculturales. Constatar y documentar la "perturbación" racial en el sistema escolar y el desplazamiento del dinero como símbolo de distinción social puede dar nuevas pistas para otros escenarios latinoamericanos.

En el último capítulo del libro, y ante la emergencia del debate en Bolivia en torno al racismo, Rea Campos identifica dos formas de explicarlo. En primer lugar, la relacionada con la lucha político-institucional y, en segundo, aquella que retoma los aspectos estructurales enfocados en la igualación del indio. La autora se apega a esta última explicación en cuanto a la estructura social como dimensión que ayuda a entender las especificidades del racismo en Oruro. En este sentido, el análisis de Rea le permite señalar que las clases medias orureñas, definidas como no indígenas, atraviesan por un estancamiento económico, mientras que algunos sectores aymaras muestran un movimiento ascendente en la estructura social.

El hecho de que la figura social del "indio" se haya desplazado en la estructura social se debe a efectos no esperados de las políticas neoliberales de los noventa y a los cambios demográficos y educativos. Además se puede ver cómo con la crisis de los ochenta la economía en Oruru se vio transformada, dado que pasó de ser minera a comercial, lo que provocó cambios en la dinámica migratoria. La clase media con mayor nivel de educación se mudó a otras regiones del país, mientras que la población indígena se trasladó de las zonas rurales a la ciudad de Oruro. Las clases medias, a pesar de estar estancadas y empobrecidas, se han negado a perder su definición de clase media, porque "su posición social está dada por su condición étnica y su permanente apelación a sus marcas corporales en cuanto a capital racial" (p. 432). Los indios en cambio han transitado de "perdedores" a emprendedores exitosos, esto lo explica Rea mediante los conceptos de resistencia de Michel Foucault, y el de creatividad cultural de Antonio Gramsci. Aunque los prejuicios sobre el origen del capital comercial de los aymaras se dio por el narcotráfico, esto no se puede comprobar, sin embargo, sí es posible observar que esta acumulación proviene de la diversificación de sus actividades económicas, del boom del contrabando de productos de exportación: "esta acumulación del capital proviene de la capacidad de agencia y de la creatividad cultural de estos actores para aprovechar las condiciones de posibilidad" (p. 454). Todo esto da como resultado que, aunque los cholos pueden presentar una mejora en su capital económico, para la clase media blanqueada, definida no indígena y estancada en su economía, el indio sigue siendo el indio que invade lugares que no le corresponden. 
Los capítulos reseñados evocan el contexto social mexicano en cuanto a la invisibilidad de los ejes de diferenciación y exclusión de lo "otro", además de la historia contemporánea mexicana y las políticas de desindianización ocurridas en la época posrevolucionaria (Korsbaek \& Sámano, 2007). El debate sobre la autoadscripción indígena o no indígena remite asimismo a distintos temas que se estudian en México y Centroamérica, a saber: la migración étnica (Suazo, 2011). El proceso de blanqueamiento al que alude la autora remite a prácticas y discursos que se instalan como moda o como parte del sentido común. Para tal línea, el estudio de Rea permite contrastar la realidad que documenta con otras realidades latinoamericanas (Castellanos \& Landázuri, 2012).

En definitiva, el libro de Rea muestra que existen procesos de diferenciación étnica-racial y, por ende, construcciones discursivas que nombran, distinguen y separan, las cuales no son estáticas ni ahistóricas, sino que están en continua interacción con las prácticas sociales y los cambios a nivel estructural. Entender el racismo como recurso de poder y no como ideología le da una potencia analítica que pone de relieve la necesidad de introducir de manera más nítida y directa las relaciones de poder en el análisis y debate latinoamericano. El alcance teórico descansa en la concepción de que lo racial es producto de un interjuego de factores estructurales que posibilita y sitúa el análisis social desde el ángulo institucional, colectivo e individual. El Estado boliviano, las organizaciones civiles de extracción indígena y los/as mismos sujetos racializados proveen niveles de análisis que dan por resultado ricos contextos y acercamientos conceptuales para la realidad boliviana.

Como toda obra, este libro de Rea tiene limitantes. Una teórica se relaciona con el cambio social, sobre todo con una interrogante: ¿cómo interactúan los cambios en la vida cotidiana en la intersección no solo con la clase sino con el género? En el análisis contemporáneo de la realidad, a la letanía de clase y raza se le agrega una más: el género, nos dice Joan Scott (1996). Si con Michel Foucault la autora sostiene que, a partir de un largo proceso de control y biologización de los cuerpos, el Estado boliviano contribuyó a la reproducción del racismo, el rostro del biopoder es de igual forma genéricamente construido. No es igual ser hombre o mujer, o cualquier otra categoría; por tanto, los cambios sociales pasan por los que se dan en las relaciones de género y con ello por la subjetivación de las exclusiones, opresiones y trastocamientos de los sujetos racializados, y generizados.

Con todo, la lectura del libro de Rea Campos es importante para conocer una más de las dimensiones de las realidades latinoamericanas en el amplio crisol cultural y social de nuestros países; es una contribución a la formación de estudiantes, docentes e investigadores o investigadoras interesados no solo en el tema en sí mismo sino también en el manejo conceptual y metodológico. 


\section{Referencias}

Bourdieu, P. \& Passeron, J. C. (2008). La reproducción. Elementos para una teoría del sistema de enseñanza. Madrid: Popular.

Castellanos Guerrero, A. \& Landázuri Benítez, G. (2012). Racismo y otras formas de intolerancia de Norte y Sur en América Latina. México: UAM/Juan Pablos Editores.

Elías, N. (2009 [1977]). El proceso de la civilización. Investigaciones sociogéneticas y psicogéneticas. México: FCE.

Korsbaek, L. \& Sámano, M. Á. (2007). Indigenismo en México: antecedentes y actualidad. Ra Ximhai, 3(1), 195-224.

Scott, J. (1996). El género una categoría útil para el análisis histórico. En Lamas, M. (Comp.). El género: La construcción cultural de la diferencia sexual (págs. 265-302). México: PUEG-UNAM/M. Á. Porrúa.

Suazo, L. (2011). Estudio migratorio de Honduras. En Gisele L. Bonnici. G. L. \& Coria M., Elba Y. (Coords.). Estudio comparativo de la legislación y politicas migratorias en Centroamérica, México y República Dominicana (pp. 323-370). México: Sin Fronteras IAP. 